\title{
Efficacy and safety of Anluohuaxian in the treatment of patients with severe Coronavirus disease 2019- a multicenter, open label, randomized controlled study: a structured summary of a study protocol for a randomised controlled trial
}

Chi Zhang ${ }^{1}$, Jiawen $\mathrm{Li}^{1}$, Zhao Wu' ${ }^{1}$, He Wang ${ }^{2}$, Chengli Que ${ }^{3}$, Hong Zhao ${ }^{1,4^{*}}$ (D) and Guiqiang Wang ${ }^{1,4,5^{*}}$

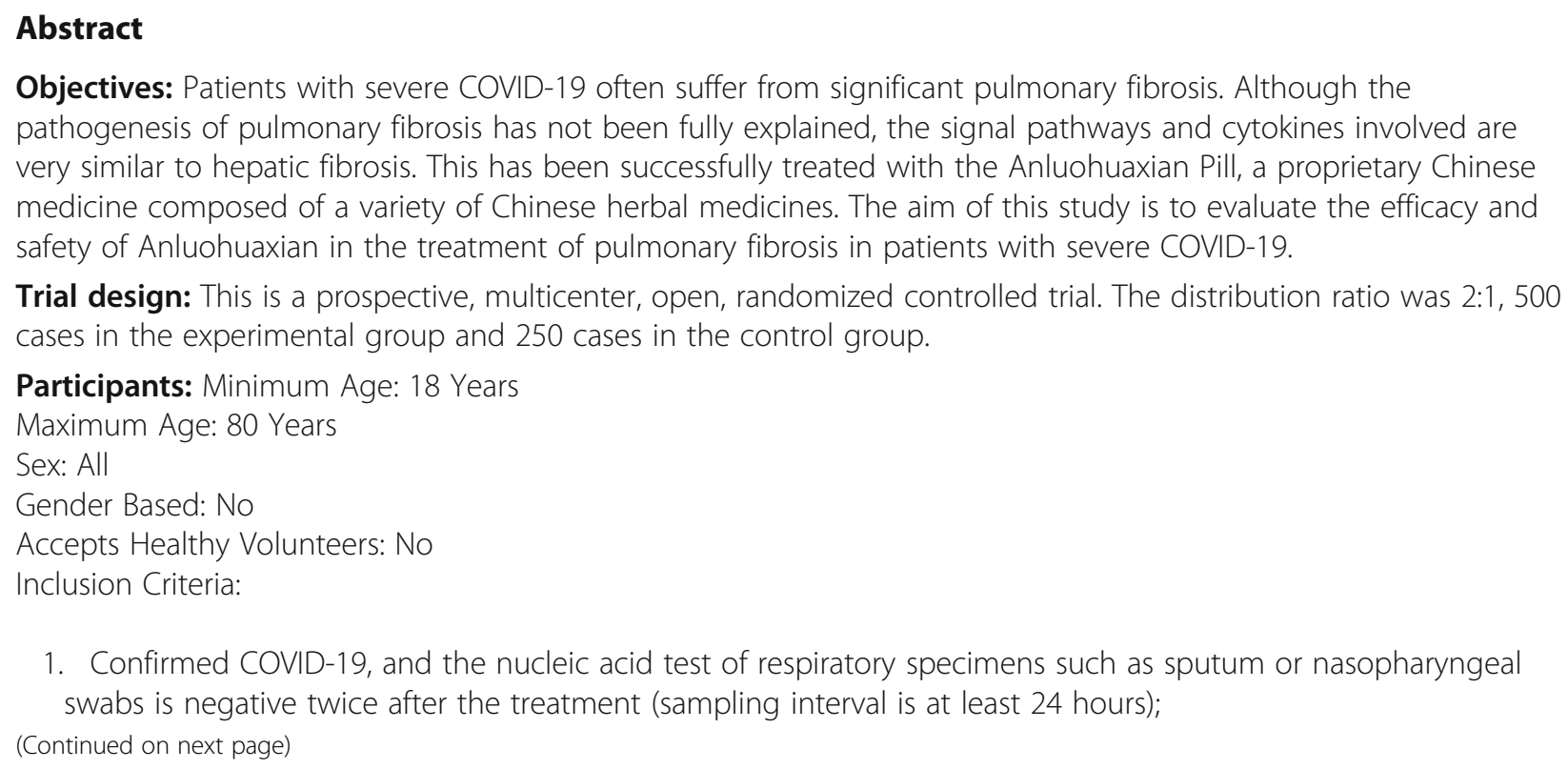

Trial design: This is a prospective, multicenter, open, randomized controlled trial. The distribution ratio was 2:1, 500 cases in the experimental group and 250 cases in the control group.

Participants: Minimum Age: 18 Years

Maximum Age: 80 Years

Sex: All

Gender Based: No

Accepts Healthy Volunteers: No

Inclusion Criteria:

1. Confirmed COVID-19, and the nucleic acid test of respiratory specimens such as sputum or nasopharyngeal swabs is negative twice after the treatment (sampling interval is at least 24 hours);

(Continued on next page)

\footnotetext{
* Correspondence: zhaohong_pufh@bjmu.edu.cn; john131212@126.com

'Department of Infectious Disease, Center for Liver Disease, Peking University

First Hospital, No.8 Xishiku Street, Xicheng District, Beijing, China

Full list of author information is available at the end of the article
}

(c) The Author(s). 2020 Open Access This article is licensed under a Creative Commons Attribution 4.0 International License, which permits use, sharing, adaptation, distribution and reproduction in any medium or format, as long as you give appropriate credit to the original author(s) and the source, provide a link to the Creative Commons licence, and indicate if changes were made. The images or other third party material in this article are included in the article's Creative Commons licence, unless indicated otherwise in a credit line to the material. If material is not included in the article's Creative Commons licence and your intended use is not permitted by statutory regulation or exceeds the permitted use, you will need to obtain permission directly from the copyright holder. To view a copy of this licence, visit http://creativecommons.org/licenses/by/4.0/. The Creative Commons Public Domain Dedication waiver (http://creativecommons.org/publicdomain/zero/1.0/) applies to the data made available in this article, unless otherwise stated in a credit line to the data. 
(Continued from previous page)

2. Negative nucleic acid test of respiratory specimens such as sputum or nasopharyngeal swabs during screening visits;

3. High-resolution CT of the lung (HRCT) indicates pulmonary fibrosis (thickness of lobular septum, honeycomb-like changes, with or without bronchial / pleural distraction);

4. Voluntarily participate in research and sign informed consent.

Exclusion Criteria:

1. Combined with severe heart, lung (diagnosed with interstitial lung disease, bronchial asthma, chronic obstructive pulmonary disease, etc.), liver and kidney disease or with endocrine, rheumatic, neurologic, malignant and other systemic diseases;

2. Have been diagnosed with connective tissue disease;

3. Pregnant or lactating women;

4. History of mental disorders, substance abuse or dependence;

5. Have used other anti-pulmonary fibrosis drugs in the past 14 days, such as nintedanib, pirfenidone, penicillamine, colchicine, tumor necrosis factor alpha blocker, imatinib, glucocorticoid hormones, morphomycodyl esters, azathioprine, cyclophosphamide, interferon- $\gamma$, and traditional Chinese medicine;

6. Researchers consider it inappropriate to participate in research;

7. Participating in other clinical research.

This mutli-centre RCT will be undertaken in 9 trial centres: The Second People's Hospital of Fuyang, Ezhou Central Hospital, Huoshenshan Hospital of Wuhan, Jinyintan Hospital of Wuhan, Tongji Hospital of Huazhong University of Science and Technology, West Hospital Union Hospital Huazhong University of Science and Technology, Wuhan Pulmonary Hospital, Zhongnan Hospital of Wuhan University, Wenzhou Medical University Affiliated First Hospital.

Intervention and comparator: The research drug is Anluohuaxian Pill, which is provided by Senlong Pharmaceutical Co., Ltd. The basic therapeutic drugs for COVID-19 involved in the study include antiviral drugs. Brands can be selected according to the treatment routines of each research center to facilitate the improvement of treatment compliance.

Main outcomes: Primary Outcome Measure:

1. Changes in high-resolution computer tomography of the lung

Changes in ground-glass shadows, interstitial or air nodules found on high-resolution computer tomography [Time Frame: 3 months]

\section{Change in 6-minute walking distance}

[Time Frame: 3 months]

Randomisation: In this study, the central randomization system (IWRS, an interactive network response system based on network) is used to randomise the groups. The subjects who met the entry criteria were randomly divided into the experimental group and the control group according to the proportion of 2:1. In this study, the block randomized grouping method is used, and the block length is 6 . The random grouping program is set up by statistical and computer professionals in the randomization process.

Blinding (masking): This is an open label trial. Trial participants, investigators, care givers, outcome assessors, and date analysts are not blinded to group assignment.

Numbers to be randomised (sample size): 750 patients are expected to be enrolled and the cases are allocated according to the ratio of 2 (Anluohuaxian combined with regular treatment group):1 (regular treatment group).

(Continued on next page) 
(Continued from previous page)

Trial Status: Protocol version number 3.0, 10th April 2020. The recruitment has not yet started.

Actual Study Start Date: April 1, 2020

Estimated Primary Completion Date: June 1, 2020

Estimated Study Completion Date: December 1, 2020

Trial registration: ClinicalTrials.gov ID: NCT04334265. Registered on 3 April 2020

Full protocol: The full protocol is attached as an additional file, accessible from the Trials website (Additional file 1). In the interest in expediting dissemination of this material, the familiar formatting has been eliminated; this Letter serves as a summary of the key elements of the full protocol.

Keywords: COVID-19, Randomised controlled trial, Protocol, Pulmonary fibrosis, Anluohuaxian

\section{Supplementary information}

Supplementary information accompanies this paper at https://doi.org/10. 1186/s13063-020-04399-8.

Additional file 1. Full study protocol.

\section{Acknowledgements}

We thank Chen Xianxiang (Wuhan Lung Hospital), Zhang Dingyu (Wuhan Jinyintan Hospital), Yu Junhua (Ezhou Central Hospital), Han Mingfeng (Fuyang second people's Hospital), Wang Xinghuan (Zhong Nan Hospital of Wuhan University), Zheng Huanwei (Shijiazhuang Fifth Hospital), Zhang Zong (Jinan Infectious Diseases Hospital), Zeng Yilan (Chengdu Public Health Clinical Center), Qiu Yuanwang (Wuxi Fifth people's Hospital), Liu Yingxia (Shenzhen third people's Hospital), Li Wei (The First Affiliated Hospital of Bengbu Medical College) for participating in this study.

\section{Authors' contributions}

$\mathrm{GW}, \mathrm{HZ}$ designed the trial. All authors read and approved the final manuscript.

\section{Funding}

This study was supported by China Mega-Project for Infectious Diseases (grant numbers 2017ZX10203202, 2013ZX10002005), China Mega-Project for Innovative Drugs (grant numbers 2016ZX09101065). The research drug, Anluohuaxian Pill, is provided by Senlong Pharmaceutical Co., Ltd The study funders had no role in study design; collection, analysis, and interpretation of data and in writing the manuscript.

\section{Availability of data and materials}

The datasets generated during and/or analyzed during the current study will be made available.

\section{Ethics approval and consent to participate}

This study was approved by the ethics committee of Peking University First Hospital on April 9, 2020, decision No. 2020-research-110. Informed consent to participate will be obtained from all participants.

\section{Consent for publication}

Not applicable.

\section{Competing interests}

The authors declare that they have no competing interests.

\section{Author details}

${ }^{1}$ Department of Infectious Disease, Center for Liver Disease, Peking University First Hospital, No.8 Xishiku Street, Xicheng District, Beijing, China.

2Department of Radiology, Peking University First Hospital, No. 8 Xishiku Street, Xicheng District, Beijing 100034, China. ${ }^{3}$ Department of Respiratory Disease, Peking University First Hospital, No. 8 Xishiku Street, Xicheng District, Beijing 100034, China. ${ }^{4}$ The Collaborative Innovation Center for Diagnosis and Treatment of Infectious Diseases, Zhejiang University, Hangzhou, Zhejiang, China. ${ }^{5}$ Peking University International Hospital, Beijing, China.
Received: 7 May 2020 Accepted: 9 May 2020

Published online: 08 June 2020

\section{Publisher's Note}

Springer Nature remains neutral with regard to jurisdictional claims in published maps and institutional affiliations.
Ready to submit your research? Choose BMC and benefit from:

- fast, convenient online submission

- thorough peer review by experienced researchers in your field

- rapid publication on acceptance

- support for research data, including large and complex data types

- gold Open Access which fosters wider collaboration and increased citations

- maximum visibility for your research: over $100 \mathrm{M}$ website views per year

At BMC, research is always in progress.

Learn more biomedcentral.com/submissions 